\title{
AN APPLICATION OF SYMBOLIC METHODS TO THE TREATMENT OF MEAN CURVATURES IN HYPERSPACE*
}

\author{
BY \\ WILLIAM HUNT BATES
}

This paper is an application of MAschkE's symbolic method for discussing invariants of quadratic differential forms, as developed in his article, A Symbolic Treatment of the Theory of Invariants of Quadratic Differential Quantics of $n$ Variables. $\dagger$ Extensive use is also made of results and methods contained in two later publications, Differential Parameters of the First Order, $\ddagger$ and The Kronecker-Gaussian Curvature of Hyperspace. § Some familiarity with these three articles is implied.

Part I of the present paper is devoted to the study of the curvatures of an $n$-space $R_{n}$ in an euclidean $(n+1)$-space $S_{n+1}$. In $\S \S 1-3$ the equations and some of the properties of the lines of curvature of $R_{n}$ in $S_{n+1}$ are developed. In particular, equation (28) gives the $n$ curvatures of the $n$ lines of curvature through a given point of $R_{n}$. The coefficients $K_{1}, \ldots, K_{n}$ of this equation are the socalled curvatures of $R_{n}$ in $S_{n+1}$, involving the coefficients $a_{i i_{i}}$ and $\alpha_{i k}$ of the two fundamental forms of $R_{n}$. With the help of his symbolic method, $\|$ MaschKe has expressed $K_{n}$, when $n$ is even, and $K_{n}^{2}$, when $n$ is odd, as rational integral functions of the coefficients $a_{i k}$ of the first fundamental form and their derivatives.

In $\S$ 4-6 similar expressions are derived for all the curvatures $K_{2 \nu}$ of even index. It does not seem possible to obtain rational results for the curvatures $K_{2 v+1}$ of odd index. In $\S 7$, however, it is shown that, with the exception of $K_{1}$, these curvatures are expressible irrationally in terms of the first fundamental quantities and their derivatives.

The symbolic expressions for $K_{2 \nu}$ and $K_{n}^{2}$ show at once that they are differential invariants of the first fundamental quadratic form for $R_{n}$, and they have meaning as invariants of any quadratic form in $n$ variables. Part II of this paper considers a space $R_{\lambda}$ defined in a space $R_{n}(n>\lambda)$, which is not neces-

* Presented to the Society December 31, 1910.

†These Transactions, vol. 4 (1903), pp. 445-469. This paper is referred to hereafter as $\mathbf{M} . \mathbf{I}$.

$\ddagger$ Ibid., vol. 7 (1906), pp. $69-80$; referred to as D. P.

\& Ibid., vol. 7 (1906), pp. $81-93$; referred to as K.-G. C.

\|In K.-G. C. 
sarily euclidean. The invariants $K_{2 \nu}$ and $K_{\lambda}^{2}$ for $R_{\lambda}$ are calculated in terms of the coefficients $a_{i k}$ belonging to the length element of $R_{n}$ and of the functions $U^{\lambda+1}, \cdots, U^{n}$ which determine the space $R_{\lambda}$ in $R_{n}$.

\section{PART I.}

Curvatures of an $n$-SPace in an $(n+1)$-space.

§1. Parametric Representation for an n-space in an $(n+1)$-space.

Let $z^{\prime}, z^{2}, \ldots, z^{n+1}$ be the coördinates* of an euclidean space $S_{n+1}$ of $n+1$ dimensions, i. e., a space whose arc-element is of the form

$$
d s^{2}=\sum_{i=1}^{n+1}\left[d z^{i}\right]^{2} .
$$

We define in $S_{n+1}$ any hypersurface, or space $R_{n}$, of $n$ dimensions, by expressing each $z$ as a funcion of $n$ independent variables $x_{1}, \cdots, x_{n}$ :

$$
\begin{gathered}
z^{\prime}=z^{\prime}\left(x_{1}, \cdots, x_{n}\right), \\
\cdot \cdot \cdot \cdot \cdot \cdot \cdot \cdot \cdot \\
z^{n+1}=z^{n+1}\left(x_{1}, \cdots, x_{n}\right) .
\end{gathered}
$$

The arc-element of $R_{n}$ is given by the equation

where

$$
d s^{2}=\sum_{i, k}^{1, \ldots, n} a_{i k} d x_{i} d x_{k}
$$

$$
a_{i k}=\sum_{j=1}^{n+1} \frac{\partial z^{j}}{\partial x_{i}} \frac{\partial z^{j}}{\partial x_{k}}=\sum_{j=1}^{n+1} z_{i}^{j} z_{k}^{j}=f_{i} f_{k},
$$

differentiation with respect to $x_{i}$ being indicated here, as in the following pages, by the lower index $i$.

A space of $\lambda$ dimensions, $\lambda<n$, would be obtained by using in (2) only $\lambda$ independent variables $x_{1}, \ldots, x_{\lambda}$. In particular, a curve in $S_{n+1}$ is obtained by expressing each $z$ as a function of one new variable $x$.

If in (2) one puts $x_{2}=\cdots=x_{n}=0$, the resulting curve is called the $x_{1}$-axis of a curvilinear system of coördinates on $R_{n}$. By letting $x_{2}, \ldots, x_{n}$ represent arbitrary constants, one gets the complete system of $x_{1}$-curves; and similarly for the other cases.

$$
\begin{aligned}
& x_{1} \text {-curves if } x_{1} \text { varies and } x_{2}, \ldots, x_{n} \text { are constants, } \\
& x_{n} \text {-curves if } x_{n} \text { varies and } x_{1}, \ldots, x_{n-1} \text { are constants. }
\end{aligned}
$$

Equations (3) and (5) give the elements of the new axes,

$$
d s_{1}^{2}=a_{11} d x_{1}^{2}, \cdots, d s_{n}^{2}=a_{n n} d x_{n}^{2},
$$

* It is assumed that no confusion will arise from writing the upper index, as MAsCHKE does, without parentheses. Exponents are only occasionally used, and will be easily recognized. 
where $d s_{k}$ is the element of the $x_{k}$-axis. Represent the direction cosines of the $x_{k}$-axis, in the old system, by $\cos \left(z^{\prime}, x_{k}\right), \cdots, \cos \left(z^{n+1}, x_{k}\right)$. Then

$$
\cos \left(z^{j}, x_{k}\right)=\frac{d z^{j}}{d s_{k}}=\frac{z_{k}^{j} d x_{k}}{\sqrt{a_{k k}} d x_{k}}=\frac{z_{k}^{j}}{\sqrt{a_{k k}}} \quad(j=1, \cdots, n+1 ; k=1, \cdots, n) .
$$

Let $\omega_{i k}$ be the angle between the $x_{i}$-axis and the $x_{k}$-axis. Then

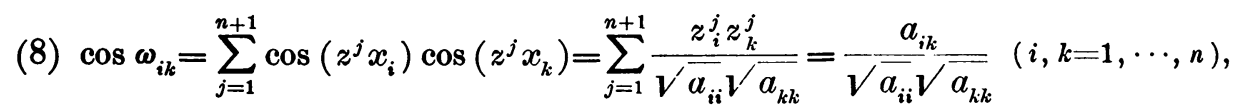
so that necessary and sufficient conditions for mutual orthogonality of the axes of the new system are

$$
a_{i k}=0 \quad(i, k=1, \cdots, n ; i \neq k) .
$$

\section{§2. General Curves on $R_{n}$.}

A general curve on $R_{n}$ may be defined by means of $n-1$ equations,

$$
U^{2}\left(x_{1}, \cdots, x_{n}\right)=\text { const., } \cdots, U^{n}\left(x_{1}, \cdots, x_{n}\right)=\text { const. }
$$

The differential equations of this curve, which we call the $U$-curve, are

$$
\sum_{i=1}^{n} U_{i}^{2} d x_{i}=0, \cdots, \sum_{i=1}^{n} U_{i}^{n} d x_{i}=0 .
$$

Its direction is defined by the ratios of $d x_{1}, \ldots, d x_{n}$ in (11). In order to solve for these differentials, let $p$ be any function of $x_{1}, \cdots, x_{n}$ which satisfies the condition *

$$
D=\left(p U^{2} \cdots U^{n}\right)=(p U) \neq 0 \text {. }
$$

If $A^{r}$ denotes the cofactor of $p_{r}$ in $D$, equations (11) are identically satisfied by

$$
d x_{1}=\rho A^{\prime}, \cdots, d x_{n}=\rho A^{n},
$$

where $\rho$ is an arbitrary parameter.

The direction cosines $\xi^{\prime}, \ldots, \xi^{n+1}$ of the $U$-curve are found as follows. From (12),

$$
\sum_{i=1}^{n} p_{i} d x_{i}=\rho \sum_{i=1}^{n} p_{i} A^{i}=\rho(p U)
$$

Then

$$
\xi^{k}=\frac{d z^{k}}{d s}=\frac{1}{d s} \sum_{i=1}^{n} z_{i}^{k} d x_{i}=\frac{\rho}{d s}\left(z^{k} U\right),
$$

where $d s$ is arc-element of the $U$-curve. Now

$$
\sum_{k=1}^{n}\left[\xi^{k}\right]^{2}=1=\sum_{k=1}^{n+1}\left[\frac{\rho}{d s}\right]^{2}\left(z^{k} U\right)^{2}=\left[\frac{\rho}{d s}\right]^{2}(f U)^{2} .
$$

\footnotetext{
* See M. I., $\$ 2$, for an explanation of this invariantive notation.
} 
Hence

$$
\frac{\rho}{d s}=\frac{1}{v \overline{(f U)^{2}}}
$$

Then the direction cosines of the $U$-curve on $R_{n}$, referred to the original system of axes, are

$$
\xi^{\prime}=\frac{\left(z^{\prime} U\right)}{\sqrt{(f U)^{2}}}, \cdots, \xi^{n+1}=\frac{\left(z^{n+1} U\right)}{\sqrt{\left(f^{\prime} U\right)^{2}}} .
$$

If there is given also a $V$-curve on $R_{n}$ by equations similar to (10), its direction cosines may be written

$$
\eta^{\prime}=\frac{\left(z^{\prime} V\right)}{\sqrt{(f V)^{2}}}, \cdots, \eta^{n+1}=\frac{\left(z^{n+1} V\right)}{V(f V)^{2}} .
$$

If $\omega$ is the angle between the two curves, we have from (14) and (15)

$$
\cos \omega=\sum_{i=1}^{n+1} \xi^{i} \eta^{i}=\sum_{i=1}^{n+1} \frac{\left(z^{i} U\right)\left(z^{i} V\right)}{\sqrt{(f U)^{2}} \sqrt{(f V)^{2}}}=\frac{(f U)(f V)}{\sqrt{(f U)^{2}} V \overline{(f V)^{2}}} .
$$

Thus a necessary and sufficient condition for orthogonality of the two curves is

$$
(f U)(f V)=0 \text {. }
$$

Equation (17) also defines the orthogonal trajectories of a system of $U$-curves on $R_{n}$. An illustration is found in the case of curves on an ordinary surface.

\section{§3. Lines of Curvature on $R$.}

A line $L$ drawn on $R_{n}$ such that the normals to $R_{n}$ along $L$ (with respect to the enclosing space $S_{n+1}$ ) generate a developable surface is called * a line of curvature of $R_{n}$ in $S_{n+1}$.

At a point $P$ of $R_{n}$ there is a unique normal to $R_{n}$ in $S_{n+1}$. Let the direction cosines of this normal be $\zeta^{\prime}, \ldots, \zeta^{n+1}$. Choose $P$ as origin of the system of $x$-axes on $R_{n}$. Then, since the normal to $R_{n}$ at $P$ is orthogonal to every direction on $R_{n}$ at $P$, we have from (7)

$$
\sum_{i=1}^{n+1} \zeta^{i} z_{k}^{i}=0 \quad(k=1, \cdots, n) .
$$

The coefficients $a_{i k}$ of the first fundamental form of $R_{n}$, given in (3) are the first fundamental quantities. The second fundamental quantities are defined by the equations

$$
\alpha_{i k}=\sum_{j=1}^{n+1} \zeta^{j} z_{i k}^{j} \quad(i, k=1, \cdots, n) .
$$

* Cf. BranchI, Lezioni di Geometria Differenziale, vol. I, p. 125. 
By differentiating (18), one obtains

$$
\alpha_{i k}=\sum_{j=1}^{n+1} \zeta^{j} z_{i k}^{j}=-\sum_{j=1}^{n+1} \zeta_{i}^{j} z_{k}^{j} \quad(i, k=1 ; \cdots, n) .
$$

The letters $g$ and $\gamma$ are to be used in this paper as symbols of the second fundamental quantities

$$
\alpha_{i k}=g_{i} g_{k}=\gamma_{i} \gamma_{k}=\alpha_{k i}
$$

Let $C$ be the curve of $S_{n+1}$ which is the envelope of the normals along $L$. Let $M\left(z^{\prime}, \cdots, z^{n+1}\right)$ be any point of $L$, and $\bar{M}\left(\bar{z}^{\prime}, \cdots, \bar{z}^{n+1}\right)$ be the point where the normal at $M$ meets $C$. Denote by $r$ the distance $M \bar{M}$, which is positive or negative according to the direction of $\bar{M}$ from $M$. Then

$$
\bar{z}^{\prime}=z^{\prime}-r \zeta^{\prime}, \cdots, \bar{z}^{n+1}=z^{n+1}-r \zeta^{n+1}
$$

Take derivatives of equations (21) with respect to the arc $s$ of $L$. Then, since $C$ is envelope of the normals along $L$,

$$
\begin{aligned}
& \frac{d \bar{z}^{\prime}}{d s}=\frac{d z^{\prime}}{d s}-r \frac{d \zeta^{\prime}}{d s}-\zeta^{\prime} \frac{d r}{d s}=q \zeta^{\prime}, \\
& \frac{d \bar{z}^{n+1}}{d s}=\frac{d z^{n+1}}{d s}-r \frac{d z^{n+1}}{d s}-\zeta^{n+1} \frac{d r}{d s}=q \zeta^{n+1},
\end{aligned}
$$

where $q$ is a factor of proportionality to be determined. Multiply equations (22) by $\zeta^{\prime}, \ldots, \zeta^{n+1}$ in order and add. We get

$$
q \sum_{i=1}^{n+1}\left[\zeta^{i}\right]^{2}=\sum_{i=1}^{n+1} \zeta^{i} \frac{d z^{i}}{d s}-r \sum_{i=1}^{n+1} \zeta^{i} \frac{d \zeta^{i}}{d s}-\frac{d r}{d s} \sum_{i=1}^{n+1}\left[\zeta^{i}\right]^{2} .
$$

Now

$$
\sum_{i=1}^{n+1}\left[\zeta^{i}\right]^{2}=1, \quad \sum_{i=1}^{n+1} \zeta^{i} \frac{d \zeta^{i}}{d s}=0, \quad \sum_{i=1}^{n+1} \zeta^{i} \frac{d z^{i}}{d s}=0
$$

since $\zeta^{\prime}, \ldots, \zeta^{n+1}$ are direction cosines of the normal and $d z^{\prime} / d s, \ldots, d z^{n+1} / d s$ are direction cosines of $L$. Substituting these results in (23), one obtains

$$
q=-\frac{d r}{d s}
$$

Then equations (22) give

$$
\frac{d z^{\prime}}{d s}=r \frac{d \zeta^{\prime}}{d s}, \cdots, \frac{d z^{n+1}}{d s}=r \frac{d \zeta^{n+1}}{d s}
$$

or

$$
\frac{d z^{\prime}}{d \zeta^{\prime}}=\frac{d z^{2}}{d \zeta^{2}}=\cdots=\frac{d z^{n+1}}{d \zeta^{n+1}}=r
$$


This result is expressed in curvilinear coördinates as follows :

$$
\begin{aligned}
z_{1}^{\prime} d x_{1}+\cdots+z_{n}^{\prime} d x_{n} & =r\left(\zeta_{1}^{\prime} d x_{1}+\cdots+\zeta_{n}^{\prime} d x_{n}\right) \\
\cdot & \cdot \cdot \cdot \cdot \cdot \cdot \cdot \cdot \\
z_{1}^{n+1} d x_{1}+\cdots+z_{n}^{n+1} d x_{n} & =r\left(\zeta_{1}^{n+1} d x_{1}+\cdots+\zeta_{n}^{n+1} d x_{n}\right)
\end{aligned}
$$

Multiply these equations in order by $z_{k}^{\prime}, \ldots, z_{k}^{n+1}$ and add. We find

$$
\sum_{i=1}^{n+1} z_{k}^{i} z_{1}^{i} d x_{1}+\cdots+\sum_{i=1}^{n+1} z_{k}^{i} z_{n}^{i} d x_{n}=r\left[\sum_{i=1}^{n+1} z_{k}^{i} \zeta_{1}^{i} d x_{1}+\cdots+\sum_{i=1}^{n+1} z_{k}^{i} \zeta_{n}^{i} d x_{n}\right]
$$

Then, by (4) and (19),

$$
a_{k 1} d x_{1}+\cdots+a_{k n} d x_{n}=-r\left[\alpha_{k 1} d x_{1}+\cdots+\alpha_{k n} d x_{n}\right] \quad(k=1, \cdots, n) .
$$

Equations (26) hold for every line of curvature on $R_{n}$. Conversely, if equations (26) be true for any curve $L$ on $R_{n}$, then there exists a curve $C$ in $S_{n+1}$ whose tangents are normal to $R_{n}$ along $L$, so that $L$ is a line of curvature on $R_{n}$ by definition.

When equations (26) are written in the form

$$
\begin{gathered}
\left(a_{11}+\alpha_{11} r\right) d x_{1}+\cdots+\left(a_{1 n}+\alpha_{1 n} r\right) d x_{n}=0, \\
\left.\cdot \cdot \cdot \cdot \cdot \cdot \cdot \cdot \cdot \cdot \cdot \cdot \cdot \cdot \cdot \cdot \cdot \cdot a_{n n}+\alpha_{n n} r\right) d x_{n}=0
\end{gathered}
$$

it is evident that the curvature $(1 / r)$ of each line of curvature through a point $P$ on $R_{n}$ must satisfy the condition

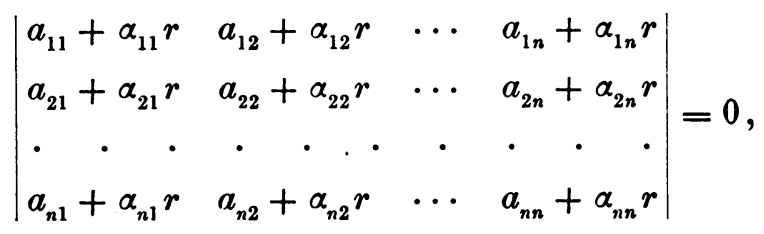

since otherwise equations (27) would have no solution except

$$
d x_{1}=d x_{2}=\cdots=d x_{n}=0 \text {. }
$$

Hence the reciprocals of the roots of (28) are exactly the curvatures of the $n$ lines of curvature through a point $P$ on $R_{n}$.

The coefficients of $r$ in (28) are called the curvatures of $R_{n}$ in $S_{n+1}$ and are discussed in $\S \S 4-7$. Before proceeding to that discussion, we derive an important property of lines of curvature.

Expressing (26) in symbolic notation, one finds

$$
f_{k} \sum_{i=1}^{n} f_{i} d x_{i}=-r g_{k} \sum_{i=1}^{n} g_{i} d x \quad(k=1, \cdots, n)
$$


If now a line of curvature be represented as a $U$-curve $(10)$, one gets from (13), and (29)

$$
f_{k}(f U)=-r g_{k}(g U) \quad(k=1, \cdots, n) .
$$

A symmetrical expression for $r$ is obtained by multiplying equations (30) in order by the cofactors of $f_{1}, \cdots, f_{n}$ in $(f U)$ and adding :

$$
r=-\frac{(f U)^{2}}{(g U)^{2}}
$$

If any two lines of curvature through $P$ be given as $U$ and $V$-curves, and their respective curvatures be denoted by $1 / r^{\prime}$ and $1 / r^{\prime \prime}$, one gets from (30)

$$
\begin{aligned}
& g_{1}(g U)=-\frac{1}{r^{\prime}} f_{1}(f U), \cdots, g_{n}(g U)=-\frac{1}{r^{\prime}} f_{n}(f U), \\
& g_{1}(g V)=-\frac{1}{r^{\prime \prime}} f_{1}(f V), \cdots, g_{n}(g V)=-\frac{1}{r^{\prime \prime}} f_{n}(f V) .
\end{aligned}
$$

Multiply the equations of the first line in order by the cofactors of $f_{1}, \cdots, f_{n}$ in $(f V)$ and add. Also multiply the equations of the second line in order by the cofactors of $f_{1}, \cdots, f_{n}$ in $(f U)$ and add. Then

$$
(g U)(g V)=-\frac{1}{r^{\prime}}(f U)(f V)=-\frac{1}{r^{\prime \prime}}(f U)(f V),
$$

so that either $r^{\prime}=r^{\prime \prime}$ or $(f U)(f V)=0$. Hence by (17) we have

Theorem I. Any two distinct lines of curvature through an ordinary point $P$ of $R_{n}$ are orthogonal to each other.

If the lines of curvatire through $P$ be taken as parameter lines, then, by $(9)$.

$$
a_{i k}=0
$$

It follows at once from (26) that also

$$
\alpha_{i k}=0 \quad(i, k=1, \cdots, n ; i \neq k) .
$$

Theorem II. If the lines of curvature at an ordinary (not umbilic) point of $R_{n}$ be taken as parameter lines, then

$$
a_{i k}=0, \quad \alpha_{i k}=0 \quad(i, k=1, \cdots, n ; i \neq k) .
$$

§4. Definition of the Curvatures of $R_{n}$ in $S_{n+1}$.

Equation (28) may be written in the form

$$
H_{0}+H_{1} r+\cdots+H_{n-1} r^{n-1}+H_{n} r^{n}=0,
$$

where *

* M. I., (9).

$$
H_{0}=\left|a_{i k}\right|=1 / \beta^{2}, \quad H_{n}=\left|\alpha_{i k}\right|
$$


while for $j=1, \cdots, n, H_{j}$ is the sum of all the determinants obtained from $\left|a_{i k}\right|$ by replacing in all possible ways $j$ columns of $\left|a_{i k}\right|$ by the corresponding columns of $\left|\alpha_{i k}\right|$. Dividing (32) by $H_{0}$ one obtains

$$
1+K_{1} r+\cdots+K_{n-1} r^{n-1}+K_{n} r^{n}=0 .
$$

The coefficient $K_{n}$ (the product of all the curvatures) is the Kronecker-Gaussian curvature of hyperspace. It has been shown to be expressible in terms of the first fundamental quantities and their derivatives (cf. K-G. C.). In this paper the coefficients of (33) are called the $n$ curvatures of $R_{n}$ in $S_{n+1}$. By definition

$$
\begin{aligned}
& K_{1}=\beta^{2} \sum_{i, k}^{1, \ldots,{ }^{n}} \alpha_{i k} A_{k}^{i}, \quad K_{2}=\beta^{2} \sum_{i_{1} i_{2}, k_{1} k_{2}}^{1, \ldots,{ }^{n}}\left|\begin{array}{c}
\alpha_{i_{1} k_{1}} \alpha_{i_{1} k_{2}} \\
\alpha_{i_{2} k_{1}} \alpha_{i_{2} k_{2}}
\end{array}\right| \cdot A_{k_{1} k_{2}}^{i_{1} i_{2}}=\sum_{i_{1} i_{2} k_{1} k_{2}}^{1, \ldots,{ }^{n}} \Delta_{i_{2} i_{2} k_{2}} A_{k_{1} k_{2}}^{i_{1} i_{2}}, \ldots, \\
& K_{m}=\beta^{2} \sum_{i_{1} \ldots i_{m} k_{1} \ldots k_{m}}^{1, \ldots, n} \Delta_{\substack{i_{1} \ldots i_{m} \\
k_{1} \ldots i_{m}}} \cdot A_{k_{1} \ldots k_{m}}^{i_{1} \ldots i_{m}} \quad \quad(m=1, \cdots, n),
\end{aligned}
$$

where $A_{k}^{i}$ is the cofactor of $a_{i k}$ in $\left|a_{i k}\right|, A_{k_{1} k_{2}}^{i_{1} i_{2}}$ is the algebraic complement of

$$
\left|\begin{array}{c}
a_{i_{1} k_{1}} a_{i_{1} k_{2}} \\
a_{i_{2} k_{1}} a_{i_{2} k_{2}}
\end{array}\right|
$$

in $\left|a_{i k}\right|$, while $\Delta_{\substack{i_{1} i_{2} \\ k_{1} k_{2}}}$ is the second minor of $\left|\alpha_{i k}\right|$ indicated for $K_{2}$ above; and similarly for the $A$ 's and $\Delta$ 's in $K_{m}$. Both sets $i_{1}, \ldots, i_{m}$ and $k_{1}, \ldots, k_{m}$ are considered as being in ascending numerical order.

\section{§5. Invariant Symbolic Forms of $K_{1}, \ldots, K_{n}$.}

If $F_{k}^{i}$ be the cofactor of $f_{k}^{i}$ in the functional determinant $\left\{f^{\prime}, \cdots, f^{n}\right\}$, Maschke* has shown that

Thus

$$
A_{k}^{i}=\frac{1}{(n-1) !} F_{i}^{\prime} F_{k}^{\prime} .
$$

$$
\begin{aligned}
K_{1}=\beta^{2} \sum_{i, k}^{i, \ldots n} \alpha_{i k} A_{k}^{i} & =\frac{\beta^{2}}{(n-1) !} \sum_{i, k}^{i, \ldots n} g_{i} g_{k} F_{i}^{\prime} F_{k}^{\prime} \\
& =\frac{\beta^{2}}{(n-1) !}\left\{g f^{2} \cdots f^{n}\right\}^{2}=\frac{1}{(n-1) !}(g f)^{2} .
\end{aligned}
$$

This suggests a method for reducing all the curvatures to convenient invariant forms. Let $F_{i_{1} \ldots i_{m}}^{1} \ldots$ be the algebraic complement of

* M. I., p. 450 .

$$
\left|\begin{array}{c}
f_{i_{1}}^{\prime} \cdots f_{i_{m}}^{\prime} \\
\cdot \cdot \cdots \cdot \cdot \\
f_{i_{1}}^{m} \cdots \cdot f_{i_{m}}^{m}
\end{array}\right|
$$


in $\left\{f^{\prime} \cdots f^{n}\right\}$. Then the product $F_{i_{1} \ldots i_{m}}^{1 \ldots} \cdot F_{k_{1} \ldots k_{m}}^{1 \ldots \ldots y}$ may be written

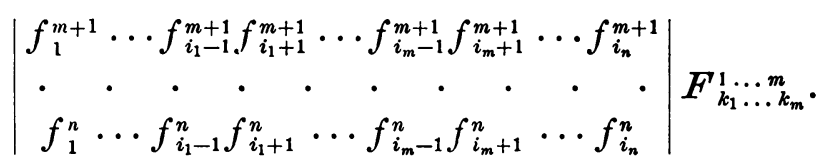

If the first determinant of this product be expanded, one finds $(n-m)$ ! terms of the form

$$
(-1)^{\mu} f_{1} \cdots f_{i_{1}-1} f_{i_{1}+1} \cdots f_{i_{m}-1} f_{i_{m}+1} \cdots f_{n} \cdot F_{k_{1} \ldots k_{m}}^{1 \ldots m}
$$

where the suppressed upper indices of the first factor are understood to be any permutation of the numbers $m+1, \cdots, n$, while $\mu$ represents the number of inversions in the permutation. Since the equivalent symbols $f^{m+1}, \cdots, f^{n}$ may be interchanged in all possible ways without altering the value of the term, let them be so interchanged for each term as to reduce the first factor to $(-1)^{\mu}$ times the principal diagonal term of $F_{i_{1} \ldots i_{m}}^{1 \ldots m}$. This causes an interchange of rows in the second (determinant) factor $F^{1}{ }_{k_{1}}^{1 \ldots m} k_{m}$ so that it becomes in each case $(-1)^{\mu}$ times its original form. Hence the above product becomes

$$
(n-m) ! f_{1}^{m+1} \cdots f_{i_{1}-1}^{i_{1}+m-1} f_{i_{1}+1}^{i_{1}+m} \cdots f_{i_{m}-1}^{i_{m}} f_{i_{m}+1}^{i_{m}+1} \cdots f_{n}^{n} \cdot F_{k_{1} \ldots k_{m}}^{1} \ldots
$$

Multiplying each $f$ into the corresponding row of the determinant $F_{k_{1} \ldots k_{m}}^{1 \ldots m}$ (which has a form similar to that given above for $F_{i_{1} \ldots i_{m}}^{1} \ldots$, we have

or

$$
F_{i_{1} \ldots i_{m}}^{1} \ldots F_{k_{1} \ldots k_{m}}^{1} \ldots k_{m}=(n-m) ! A_{k_{1} \ldots k_{m}}^{i_{1} \ldots i_{m}}
$$

$$
A_{k_{1} \ldots k_{m}}^{i_{1} \ldots i_{m}}=\frac{1}{(n-m) !} F_{i_{1} \ldots i_{m}}^{1 \ldots m} \cdot F_{k_{1} \ldots k_{m}}^{1 \ldots}
$$

Also

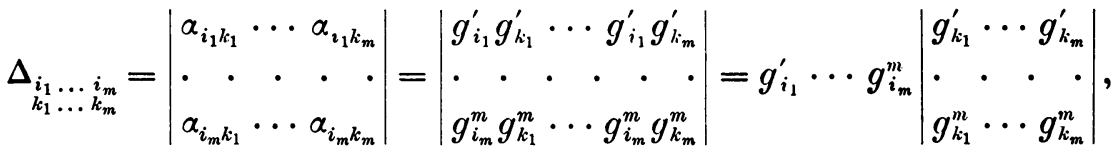

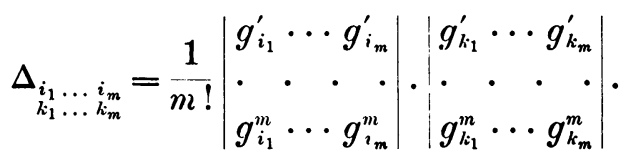

Substituting (35) and (36) in (34), one finds, by a well-known theorem of determinants,

$$
K_{m}=\frac{1}{m !(n-m) !}\left(g^{\prime} \cdots g^{m} f^{m+1} \cdots f^{n}\right)^{2}=\frac{1}{m !(n-m) !}\left(g^{\prime} \cdots g^{m} f\right)^{2} .
$$

In particular,

$$
K_{1}=\frac{1}{(n-1) !}(g f)^{2}=\Delta_{1} g
$$

where, by M. I. (22), $\Delta_{1} g$ is the first differential parameter of the first quadratic form $(\cdot \dot{)})$. 
Since the other coefficients are corresponding differential parameters (the number of $g$ 's being the same as the subscript of $K$ ), it would seem fitting to generalize the notation and set*

$$
K_{m}=\frac{1}{m !(n-m) !}\left(g^{\prime} \cdots g^{m} f\right)^{2}=\Delta^{m} g
$$

with the note that $\Delta^{\prime} g=\Delta_{1} g$.

\section{§6. Expression of $K_{2 \nu}$ in terms of the first Fundamental Quantities and Derivatives.}

The generalization of the Gauss equation shows that any second order deter minant of the second fundamental quantities is equal to a Riemann quadruple index symbol, which is expressible in terms of the first fundamental quantities

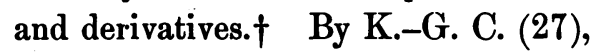

$$
\left|\begin{array}{c}
\alpha_{i_{1} k_{1}} \alpha_{i_{1} k_{2}} \\
\alpha_{i_{2} k_{1}} \alpha_{i_{2} k_{2}}
\end{array}\right|=\left(i_{1} i_{2} k_{1} k_{2}\right)=\frac{1}{(n-1) !} f_{i_{1}}^{\prime} f_{i_{2}}^{2}\left|\begin{array}{l}
(f a)_{k_{1}}^{\prime}(f a)_{k_{2}}^{\prime} \\
(f a)_{k_{1}}^{2}(f a)_{k_{2}}^{2}
\end{array}\right| .
$$

By an easy induction, any even order determinant of the $\alpha$ 's is expressed in terms of the symbols of the $a$ 's as follows: $\ddagger$

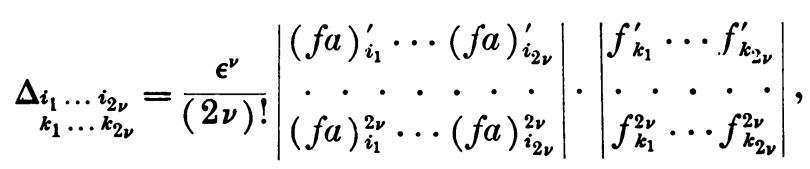

where $\epsilon=1 /(n-1) ! ;$ the symbol $(f a)^{j}$ contains $f^{j}$ and $a^{2} \ldots a^{n}$, while the symbols $a$ in every consecutive pair $\left(f a^{2} \cdots a^{n}\right)^{2 \lambda-1},\left(f a^{2} \cdots a^{n}\right)^{2 \lambda}$ are equal when they have the same index, otherwise they are distinct but equivalent symbols of the first fundamental form (3).

Now from (34), (35), and (39),

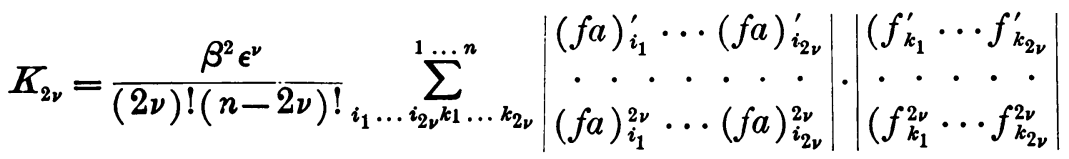

$$
\begin{aligned}
& \times F_{i_{1} \ldots i_{2 \nu}}^{1} \mathrm{~F}_{k_{1} \ldots k_{2 \nu}}^{1 \ldots 2 \nu},
\end{aligned}
$$

or

$$
K_{2 \nu}=\frac{\epsilon^{\nu}}{(2 \nu) !(n-2 \nu) !}\left((f a)^{\prime} \cdots(f a)^{2 \nu} f\right)(f) .
$$

* The use of $\Delta_{m} g$ would conflict with the second differential parameter of ordinary differential geometry, which has an entirely different meaning. Cf. BranchI, Lezioni di Geometria Differenziale, vol. I, p. 67.

t.M. I., (117)-(126).

† Cf. K. -G. C., (28). 
This gives Maschke's expression * for $K_{n}$ when $n$ is even :

$$
K_{n}=\frac{1}{n ![(n-1) !]^{n / 2}}\left((f a)^{\prime} \cdots(f a)^{n}\right)(f) \quad(n \text { even }) .
$$

Theorem. The mean curvatures $K_{2 \nu}$, with even subscript, are represented in (40) as rational integral functions of the coefficients of the first fundamental form and their derivatives.

\section{§7. Expression of $K_{2 v+1}$ in terms of the first Fundamental Quantities} and Derivatives, when $\nu$ is greater than zero.

Use is made of the determinant theorem

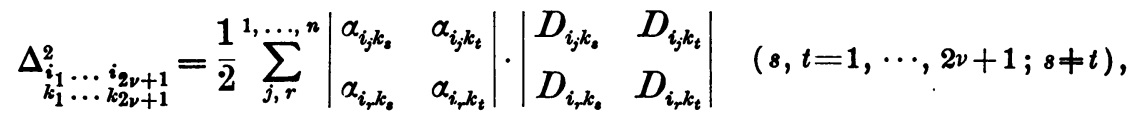

where $\nu \neq 0$ and the $D$ 's are cofactors of the corresponding $\alpha$ 's in $\Delta_{k_{1}}^{i_{1}} \ldots i_{2 \nu+1}^{i_{2 \nu+1}}$ and are therefore all of even order and expressible by (39). The results are

$$
\begin{array}{ll}
D_{i_{j} k_{s}}=\frac{\epsilon^{\nu}}{(2 \nu) !} F_{i_{j}}^{\prime \prime}(F A)_{k_{s}}^{\prime}, & D_{i_{j} k_{t}}=\frac{\epsilon^{\nu}}{(2 \nu) !} \Phi_{i_{j}}^{\prime}(\Phi B)_{k_{t}}^{\prime}, \\
D_{i_{r} k_{s}}=\frac{\epsilon^{\nu}}{(2 \nu) !} F_{i_{r}}^{\prime}(F A)_{k_{s}}^{\prime}, & D_{i_{r} k_{t}}=\frac{\epsilon^{\nu}}{(2 \nu) !} \Phi_{i_{r}}^{\prime}(\Phi B)_{k_{t}}^{\prime},
\end{array}
$$

where $F_{i_{j}}^{\prime}$ is the cofactor of $f_{i_{j}}^{\prime}$ in $\left\{f_{i_{1}}^{\prime} \cdots f_{i_{2 \nu+1}}^{2 \nu+1}\right\}, \cdots,(\Phi B)_{k_{t}}^{\prime}$ is the cofactor of $(\phi b)_{k_{i}}^{\prime}$ in $\left\{(\phi b)_{k_{1}}^{\prime} \cdots(\phi b)_{k_{2 \nu+1}^{2 \nu+1}}^{2 \nu}\right\}$ Also, by M. I. (120),

$$
\left|\begin{array}{cc}
\alpha_{i_{j} k_{s}} & \alpha_{i_{j} k_{t}} \\
\alpha_{i, k_{s}} & \alpha_{i, k_{t}}
\end{array}\right|=\epsilon(f c)_{k_{s}}^{\prime}(\phi c)_{k_{t}}^{\prime}\left|\begin{array}{ll}
f_{i_{j}}^{\prime} & \phi_{i_{j}}^{\prime} \\
f_{i_{r}}^{\prime} & \phi_{i_{r}}^{\prime}
\end{array}\right|
$$

Substituting in (41), we find

$$
\begin{aligned}
& \Delta_{\substack{i_{1} \\
k_{1} \ldots i_{z_{2}+1}}}^{2}=\frac{\epsilon^{2 \nu+1}}{[(2 \nu) !]^{2}}(f c)_{k_{s}}^{\prime}(F A)_{k_{s}}^{\prime}(\phi c)_{k_{t}}^{\prime}(\Phi B)_{k_{t}}^{\prime} \\
& \times \frac{1}{2} \sum_{j, r}^{1} \sum^{2 \nu+1}\left|\begin{array}{ll}
f_{i_{j}}^{\prime} & \phi_{i_{j}}^{\prime} \\
f_{i_{r}}^{\prime} & \phi_{i_{r}}^{\prime}
\end{array}\right| \cdot\left|\begin{array}{cc}
F_{i_{j}}^{\prime} & \Phi_{i_{j}}^{\prime} \\
F_{i_{r}}^{\prime} & \Phi_{i_{r}}^{\prime}
\end{array}\right| .
\end{aligned}
$$

This last sum expands into

$$
\begin{aligned}
& \overline{2} \sum_{j, r}^{1, \ldots, 2 \nu+1}\left[f_{i_{j}}^{\prime} F_{i_{j}}^{\prime} \phi_{i_{r}}^{\prime} \Phi_{i_{r}}^{\prime}-f_{i_{j}}^{\prime} \Phi_{i_{j}}^{\prime} \phi_{i_{r}}^{\prime} F_{i_{r}}^{\prime}-f_{i_{r}}^{\prime} \Phi_{i_{r}}^{\prime} \phi_{i_{j}}^{\prime} F_{i_{j}}^{\prime}+f_{i_{r}}^{\prime} F_{i_{r}}^{\prime} \phi_{i_{j}}^{\prime} \Phi_{i_{j}}^{\prime}\right] \\
& =\left|\begin{array}{lr}
\left\{f_{i_{1}}^{\prime} \cdots f_{i_{2 \nu+1}}^{2 v+1}\right\} & \left\{f_{i_{1}}^{\prime} \phi_{i_{2}}^{2} \cdots \phi_{i_{2 \nu+1}}^{2 v+1}\right\} \\
\left\{\phi_{i_{1}}^{\prime} f_{i_{2}}^{2} \cdots f_{i_{2 \nu+1}}^{2 \nu+1}\right\} & \left\{\phi_{i_{1}}^{\prime} \cdots \phi_{i_{2 \nu+1}}^{2 \nu+1}\right.
\end{array}\right|,
\end{aligned}
$$

so that

$$
\begin{aligned}
& \underset{\substack{i_{1} \ldots i_{1} \\
k_{1} \ldots k_{2 \nu+1}}}{\Delta_{2 \nu+1}^{2}}=\frac{\epsilon^{2 \nu+1}}{[(2 \nu) !]^{2}}(f c)_{k_{\mathrm{s}}}^{\prime}(F A)_{k_{s}}^{\prime}(\phi c)_{k_{t}}^{\prime}(\Phi B)_{k_{t}}^{\prime} \\
& \times\left|\begin{array}{lr}
\left\{f_{i_{1}}^{\prime} \cdots f_{i_{2 \nu+1}}^{2 \nu+1}\right\} & \left\{f_{i_{1}}^{\prime} \phi_{i_{2}}^{2} \cdots \phi_{i_{2 \nu+1}}^{2 \nu+1}\right\} \\
\left\{\phi_{i_{1}}^{\prime} f_{i_{2}}^{2} \cdots f_{i_{2 \nu+1}}^{2 \nu+1}\right\} & \left\{\phi_{i_{1}}^{\prime} \cdots \phi_{i_{2 \nu+1}}^{2 \nu+1}\right.
\end{array}\right| .
\end{aligned}
$$

* K.-G. C., (29). 
By (41) this equation holds for all values of $s$ and $t$ from 1 to $2 \nu+1$ except. $s=t$. When $s=t$, the second member vanishes. Sum the equations given by using all values of $s$ and $t$ from 1 to $2 \nu+1$ and divide by $(2 \nu+1) 2 \nu$; also multiply by $\beta^{4}$. Then

$$
\begin{aligned}
& \beta^{4} \Delta_{\substack{i_{1} \ldots i_{2 \nu+1} \\
k_{1} \ldots k_{2 \nu+1}}}^{2}=\frac{\varepsilon^{2 \nu+1}}{(2 \nu+1)(2 \nu)[(2 \nu) !]^{2}}\left((f c)_{k_{1}}^{\prime}(f a)_{k_{2}}^{2} \cdots(f a)_{k_{2 \nu+1}}^{2 \nu+1}\right) \\
& \times\left((\phi c)_{k_{1}}^{\prime}(\phi b)_{k_{2}}^{2} \cdots(\phi b)_{k_{i_{2 \nu}+1}^{2 \nu+1}}^{2 \nu}\right)\left|\begin{array}{ll}
\left\{f_{i_{1}}^{\prime} \cdots f_{i_{2 \nu}+1}^{2+1}\right\} & \left\{f_{i_{1}}^{\prime} \phi_{i_{2}}^{2} \cdots \phi_{i_{2 \nu}+1}^{2 \nu+1}\right\} \\
\left\{\phi_{i_{1}}^{\prime} f_{i_{2}}^{2} \cdots f_{i_{2 \nu}}^{2 \nu+1}\right\} & \left\{\phi_{i_{1}}^{\prime} \cdots \phi_{i_{2 \nu+1}}^{2 \nu+1}\right\}
\end{array}\right| .
\end{aligned}
$$

And by (34)

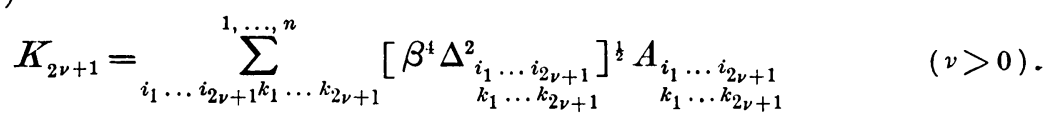

Thus by (34) and (42) we have $K_{2 \nu+1}(\nu>0)$ expressed in terms of the first fundamental quantities and derivatives (but only in the irrational form of a sum of square roots).

The case of $K_{1}$ presents special difficulty :

$$
K_{1}=\beta^{2} \sum_{i k}^{1, \ldots n} \alpha_{i k} A_{k}^{i}
$$

In K.-G. C. (p. 24), Maschke suggests a method for expressing the $\alpha$ 's in terms of the $a$ 's when $n$ is odd. His formula (24) should, however, be written,

$$
\alpha_{11} \Delta^{n-2}=\left|\begin{array}{ccc}
A_{22} \cdots & A_{2 n} \\
A_{n 2} \cdots & A_{n n}
\end{array}\right|
$$

If $n$ is odd, the elements of the second member of (43) are of even order, and therefore expressible by (39), and similarly for every $\alpha$. But $\Delta$ itself is of odd order, and is raised to an odd power $(n-2$ instead of $n-1) .{ }^{*} \quad$ Equation (43) is true also for even values of $n$, so that the $\alpha$ 's are always expressible by (43) in terms of the first fundamental quantities and derivatives (if $n>2$ ), but in all cases irrationally.

Using (43), the author has calculated irrational values of $K_{1}$ when $n$ is greater than two ; but the notation is so complicated that the presentation of the results seems impracticable, if not also useless. $\dagger$

If $2 \nu+1=n$, the sum reduces to a single term and formulas (34) and (42)

* Cf. BûCHER, Introduction to Higher Algebra, §11.

$\dagger$ In a recent paper the author has calculated the value of $K_{1}$ as well as of the other curvatures of odd subsoript, for a space of $n-1$ dimensions defined in $R_{n}$ by the equation $U\left(x_{1} \cdots x_{n}\right)=0$. These values involve only the coefficients of the first fundamental form of $R_{n}$ and their derivatives, together with the function $U$. 
give a rational value for $K_{n}^{2}$,

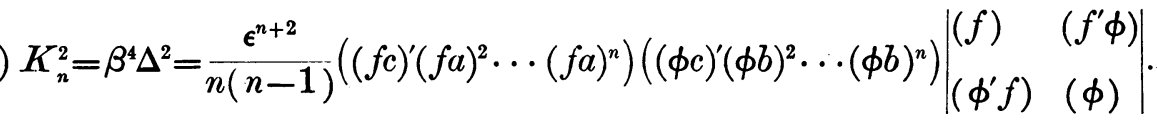

By the method used in K.-G. C. (p. 86), this may be reduced to Maschke's form $(31)$ : *

$$
K_{n}^{2}=\frac{1}{n[(n-1) !]^{n+2}}\left((f c)^{\prime}(f a)^{2} \cdots(f a)^{n}\right)\left((\phi c)^{\prime}(\phi b)^{2} \cdots(\phi b)^{n}\right)\left(f^{\prime} \phi^{\prime} f\right)\left(f^{2} \phi^{2} \phi\right) .
$$

The rather unsatisfactory results of this section are then as follows:

If $n$ is odd, $K_{n}^{2}$ is expressed by (45) as a rational function of the first fundamental quantities and their derivatives. Equations (34) and (42) give irrational expressions for the curvatures of odd index except $K_{1}$, for which no expression is here given.

\section{PART II.}

\section{INVARIANTS OF $R_{\lambda}$ IN $R_{n}$.}

The quantities $K_{2 \nu}$ and $K_{n}^{2}$, for $n$ odd, are by their forms (40) and (45) differential invariants of the first fundamental quadratic form (3). When (3) defines the arc-element of a space $R_{n}$ of $n$ dimensions contained in an euclidean space $S_{n+1}$ of $n+1$ dimensions, these $K$ 's have the geometric meaning already assigned to them. It is our object $\dagger$ to find corresponding invariants of a space $R_{\lambda}$ of $\lambda$ dimensions, represented as differential parameters of a general space $R_{n}$ of higher dimensions containing $R_{\lambda}$.

\section{§1. Definitions and Preliminary Formulas.}

In the general space $R_{n}$, of $n$ dimensions, whose coördinates are $x_{1}, \ldots, x_{n}$ and whose arc-element is defined by equation (3), let the space $R_{\lambda}$ of $\lambda$ dimensions $(\lambda<n)$ be defined by the $n-\lambda$ equations

$$
U^{\lambda+1}\left(x_{1}, \cdots, x_{n}\right)=\text { const., } \cdots, U^{n}\left(x_{1}, \cdots, x_{n}\right)=\text { const. }
$$

If $\lambda$ other arbitrarily chosen functions of $x_{1}, \cdots, x_{n}$, say $u^{\prime}, \cdots, u^{\lambda}$, such that

$$
\Delta=\left(u^{\prime} \cdots u^{\lambda} U^{\lambda+1} \cdots U^{n}\right) \neq 0,
$$

are adjoined to these, the space $R_{\lambda}$ may also be represented in parametric form

$$
x_{1}=x_{1}\left(u^{\prime}, \cdots, u^{\lambda}\right), \cdots, x_{n}=x_{n}\left(u^{\prime}, \cdots, u^{\lambda}\right),
$$

* In MASCHKE's reduction there are two slight numerical errors which balance each other. His. equation (30) differs from (44) above in that he has divided by $n^{2}$ instead of by $n(n-1)$; while in his reduction of (30) there are $n-1$ of the terms which become equal, instead of $n$.

† Cf. K.-G. C., \& 5 . 
by solving the $n-\lambda$ equations (46) with the $\lambda$ equations

$$
u^{\prime}\left(x_{1}, \cdots, x_{n}\right)=u^{\prime}, \cdots, u^{\lambda}\left(x_{1}, \cdots, x_{n}\right)=u^{\lambda} .
$$

Any $n$ differentials satisfying the $n-\lambda$ equations, found by differentiating (46),

$$
\sum_{i=1}^{n} U_{i}^{\lambda+1} d x_{i}=0, \cdots, \quad \sum_{i=1}^{n} U_{i}^{n} d x_{i}=0
$$

determine a certain direction in $R_{\lambda}$. In order to find these differentials in tems of $d u^{\prime}, \ldots, d u^{\lambda}$, we differentiate also equations (48) and solve the set

$$
\begin{aligned}
& u_{1}^{\prime} d x_{1}+\cdots+u_{n}^{\prime} d x_{n}=d u^{\prime}, \\
& u_{1}^{\lambda} d x_{1}+\cdots+u_{n}^{\lambda} d x_{n}=d u^{\lambda}, \\
& U_{1}^{\lambda+1} d x_{1}+\cdots+U_{n}^{\lambda+1} d x_{n}=0 \text {, } \\
& U_{1}^{n} d x_{1}+\cdots+U_{n}^{n} d x_{n}=0 .
\end{aligned}
$$

If $A^{k r}$ be the cofactor of $u_{r}^{k}$ in $\Delta$, then

and therefore,

$$
d x_{r}=\frac{1}{\Delta} \sum_{k=1}^{\lambda} A^{k r} \bar{d} u^{k}
$$

$$
\sum_{r=1}^{n} p_{r} d x_{r}=\frac{1}{\Delta} \sum_{k=1}^{\lambda}\left\{u^{\prime} \cdots u^{k-1} p u^{k+1} \cdots u^{\lambda} U\right\} d u^{k},
$$

where $p$ is any ordinary function of $x_{1}, \ldots, x_{n}$.

In order to find the expression for $d s$ in terms of $u^{\prime}, \ldots, u^{\lambda}$, we introduce for the differential quantic (3) the symbolic form

$$
d s^{2}=\sum_{i, k}^{1, \ldots n} a_{i k} d x_{i} d x_{k}=\left[\sum_{i=1}^{n} f_{i} d x_{i}\right]^{2} .
$$

Then (49) gives for the length element in $R_{\lambda}$

$$
\begin{aligned}
d s^{2} & =\frac{1}{\Delta^{2}}\left[\sum_{i=1}^{\lambda}\left\{u^{\prime} \cdots u^{i-1} f u^{i+1} \cdots u^{\lambda} U\right\} d u_{i}\right]^{2} \\
& =\frac{1}{\beta^{2} \Delta^{2}}\left[\sum_{i=1}^{\lambda}\left(u^{\prime} \cdots u^{i-1} f u^{i+1} \cdots u^{\lambda} U\right) d u_{i}\right]^{2} .
\end{aligned}
$$

We may also introduce for $d s^{2}$, as given in terms of $u^{\prime}, \ldots, u^{\lambda}$, the symbolic form

$$
d s^{2}=\left[\sum_{i=1}^{\lambda} \mathfrak{f}_{i} d u^{i}\right]^{2}
$$

By comparing (50) and (51) we find

(52) $\mathrm{f}_{i}=\frac{1}{\Delta}\left\{u^{\prime} \cdots u^{i-1} f u^{i+1} \cdots u^{\lambda} U\right\}=\frac{1}{\beta \Delta}\left(u^{\prime} \cdots u^{i-1} f u^{i+1} \cdots u^{\lambda} U\right)$. 
If we use the symbols of form (51), the invariants $K_{2 v}$ and $K_{\lambda}^{2}$ ( $\lambda$ odd) of $R_{\lambda}$ may be written, by (40) and (45),

$$
\begin{aligned}
& (2 \nu) !(\lambda-2 \nu) ![(\lambda-1) !]^{\nu} K_{2 \nu}=G_{2 \nu}=\left((\mathfrak{f a})^{\prime} \cdots(\mathfrak{f} \mathfrak{a})^{2 \nu} \mathfrak{f}^{2 \nu+1} \cdots \mathfrak{f}^{\lambda}\right)\left(\mathfrak{f}^{\prime} \cdots \mathfrak{f}^{\lambda}\right), \\
& \begin{array}{r}
\lambda[(\lambda-1) !]^{\lambda+2} K_{\lambda}^{2}=G_{\lambda}^{2}=\left((\mathfrak{f c})^{\prime}(\mathfrak{f a})^{2} \cdots(\mathfrak{f a})^{\lambda}\right) \\
\quad \times\left((\mathfrak{g c})^{\prime}(\mathfrak{g} b)^{2} \cdots(\mathfrak{g} b)^{\lambda}\right)\left(\mathfrak{f}^{\prime} \mathfrak{g}^{\prime} \mathfrak{f}^{3} \cdots \mathfrak{f}^{\lambda}\right)\left(\mathfrak{f}^{2} \mathfrak{g}^{2} \cdots \mathfrak{g}^{\lambda}\right),
\end{array}
\end{aligned}
$$

where $G_{2 \nu}$ and $G_{\lambda}^{2}$ are introduced merely for convenience. In all invariantive brackets containing the new symbols, of the quadratic form (51), the differentiation is with respect to the $\lambda$ variables $u^{\prime}, \ldots, u^{\lambda}$. This is indicated sufficiently by the German type and the number of symbols inside the brackets. $\beta_{u}$ is defined by the equation

$$
\left(\tilde{f}^{\prime} \cdots \dot{f}^{\lambda}\right)=\beta_{u}\left\{\mathfrak{f}^{\prime} \cdots \mathfrak{f}^{\lambda}\right\} \text {. }
$$

We now proceed to compute the values of the invariantive expressions used in (53) and (54) in terms of the symbols of the first fundamental form (3), of $R_{n}$ and the functions $U^{\lambda+1}, \ldots, U^{n}$ which define $R_{\lambda}$ in $R_{n}$.

By means of (52) and D. P. (3), we obtain

$$
\left\{\tilde{f}^{\prime} \cdots f^{\lambda}\right\}=\frac{1}{\Delta^{\lambda}}\left\{f^{\prime} \cdots f^{\lambda} U\right\}\left\{u^{\prime} \cdots u^{\lambda} U\right\}^{\lambda-1}=\frac{1}{\Delta}\left\{f^{\prime} \cdots f^{\lambda} U\right\},
$$

so that

$$
\frac{1}{\beta_{u}}\left(f^{\prime} \cdots f^{\lambda}\right)=\frac{1}{\beta \Delta}\left(f^{\prime} \cdots f^{\lambda} U\right) .
$$

To calculate the value of $\beta_{u}$, square (55) and simplify the result by placing $\left(f^{\prime} \cdots f^{\lambda}\right)^{2}=\lambda !$, according to M. I. $(17)$, and $\left(f^{\prime} \cdots f^{\lambda} U\right)^{2}=\lambda !(n-\lambda) ! \Delta^{n-\lambda} U$ by (38). This gives

$$
\beta_{u}=\omega \beta \Delta, \quad \omega=\sqrt{\frac{1}{(n-\lambda) ! \Delta^{n-\lambda} U}} .
$$

Then

$$
\left(f^{\prime} \cdots f^{\lambda}\right)=\omega\left(f^{\prime} \cdots f^{\lambda} U\right) \text {. }
$$

The other invariantive forms in (53) and (54) are reduced by the same method, and by interchanging equivalent symbols, giving*

$$
\begin{gathered}
\left(f^{\prime} \cdots f^{\lambda}\right)=\omega\left(f^{\prime} \cdots f^{\lambda} U\right), \\
\left(\mathfrak{f}^{\prime} \mathfrak{g}^{\prime} \mathfrak{f}^{3} \cdots f^{\lambda}\right)=\omega\left(f^{\prime} \phi^{\prime} f^{3} \cdots f^{\lambda} U\right), \quad\left(\mathfrak{f}^{2} \mathfrak{g}^{2} \cdots g^{\lambda}\right)=\omega\left(f^{2} \phi^{2} \cdots \phi^{\lambda} U\right) \\
\left((\mathfrak{f a})^{\prime} \cdots(\mathfrak{f a})^{2 \nu} \mathfrak{f}^{2 \nu+1} \cdots \mathfrak{f}^{\lambda}\right) \\
=\omega\left(\omega(f a U)^{\prime}, \omega(f a U)^{2}, \cdots, \omega(f a U)^{2 \nu}, f^{2 \nu+1} \cdots f^{\lambda} U\right),
\end{gathered}
$$

* Inside the invariantive brackets, we have followed MAschke's custom of omitting commas between symbols, except where ambiguity might occur. Cf. M. I., p. 448.

Trans. Am. Math. Soc. 3 


$$
\begin{aligned}
\left((\mathfrak{f c})^{\prime}(\mathfrak{f a})^{2} \cdots(\mathfrak{f a})^{\lambda}\right) & =\omega\left(\omega(f c U)^{\prime}, \omega(f a U)^{2}, \ldots, \omega(f a U)^{\lambda} U\right), \\
\left((\mathfrak{g c})^{\prime}(\mathfrak{g} \mathfrak{b})^{2} \cdots(\mathfrak{g b})^{\lambda}\right) & =\omega\left(\omega(\phi c U)^{\prime}, \omega(\phi b U)^{2}, \ldots, \omega(\phi b U)^{\lambda} U\right) .
\end{aligned}
$$

By (53) and (57),

\section{§2. Expression for $K_{2 v}$.}

$$
G_{2 \nu}=\omega^{2}\left(\omega(f a U)^{\prime}, \cdots, \omega(f a U)^{2 \nu}, f^{2 \nu+1} \cdots f^{\lambda} U\right)\left(f^{\prime} \cdots f^{\lambda} U\right) .
$$

Applying D. P. (4) to the second member, we get

$$
\begin{aligned}
& G_{2 \nu}=\omega^{2 \nu+2}\left((f a U)^{\prime} \cdots(f a U)^{2 \nu} \cdot f^{2 \nu+1} \cdots f^{\lambda} U\right)\left(f^{\prime} \cdots f^{\lambda} U\right)+\omega^{2 \nu+1}\left(f^{\prime} \cdots f^{\wedge} U\right) \\
& \times \sum_{k=1}^{2 v}(f a U)^{k}\left((f a U)^{\prime} \cdots(f a U)^{k-1}, \omega,(f a U)^{k+1} \cdots(f a U)^{2 \nu} f^{2 \nu+1} \cdots f^{\lambda} U\right) .
\end{aligned}
$$

It will now be shown that each term of this last sum vanishes. Aside from the factor $\omega^{2 \nu+1}$, each odd term of this sum may be written in the form

$$
\begin{aligned}
T=(-1)^{\lambda-1}\left(f^{k+1} \cdots f^{\lambda} f^{\prime} \cdots f^{k} U\right)(f a U)^{k} \\
\quad \times\left((f a U)^{\prime} \cdots(f a U)^{k-1}, \omega,(f a U)^{k+1} \cdots(f a U)^{2 \nu} f^{2 \nu+1} \cdots f^{\lambda} U\right) .
\end{aligned}
$$

Applying D. P. (1) to the first two brackets of the second member, we obtain

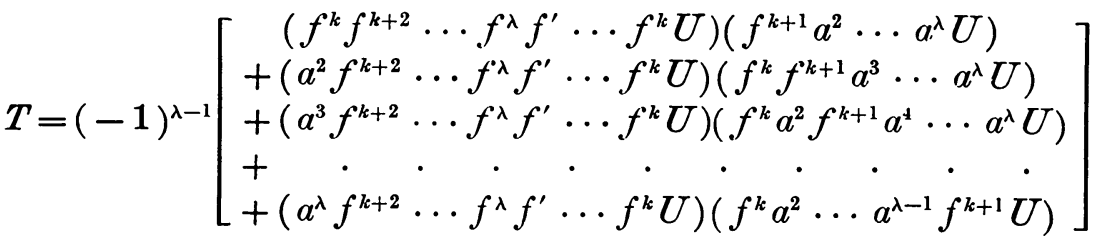

$$
\begin{aligned}
& \times\left((f a U)^{\prime} \cdots(f a U)^{k-1}, \omega,(f a U)^{k+1} \cdots(f a U)^{2 v} f^{2 v+1} \cdots f^{\lambda} U\right) \text {. }
\end{aligned}
$$

Of these $\lambda$ terms, the first vanishes because of two identical rows in the first bracket, while the others become equal to each other if we interchange $f^{k+1}$ with $a^{2} \ldots a^{\lambda}$ in turn and in each case restore the original order in $(f a U)^{k+1}$ by the interchange of two rows.* Thus

$$
\begin{aligned}
T=(-1)^{\lambda-1}(1-\lambda)\left(f^{k+1} \cdots f^{\lambda} f^{\prime} \cdots f^{k} U\right)\left(f^{k} a^{2} \cdots a^{\lambda} U\right) \\
\quad \times\left((f a U)^{\prime} \cdots(f a U)^{k-1}, \omega,(f a U)^{k+1} \cdots\right)=(-1)^{\lambda-1}(1-\lambda) T .
\end{aligned}
$$

Hence $T=0$ for odd values of $k$.

If $k$ is even, each term $T$ may be written

$$
\begin{aligned}
& T=\left(f^{k-1} \cdots f^{\lambda} f^{\prime} \cdots f^{k-2} U\right)(f a U)^{k} \\
& \quad \times\left((f a U)^{\prime} \cdots(f a U)^{k-1}, \omega,(f a U)^{k+1} \cdots(f a U)^{2 \nu} f^{2 v+1} \cdots f^{\lambda} U\right) .
\end{aligned}
$$

${ }^{*}$ Cf. K.-G. C., p. 92. 
By applying D. P. (1) to the first two brackets, and proceeding as above, one finds $T=0$ also for even values of $k$.

With the help of these results (58) becomes

$$
G_{2 \nu}=\omega^{2 \nu+2}\left((f a U)^{\prime} \cdots(f a U)^{2 \nu} f^{2 \nu+1} \cdots f^{\lambda} U\right)\left(f^{\prime} \cdots f^{\lambda} U\right) .
$$

Then, by (53) and (56),

$$
K_{2 \nu}=\frac{(\lambda-1) !\left((f a U)^{\prime} \cdots(f a U)^{2 \nu} f^{2 \nu+1} \cdots f^{\lambda} U\right)\left(f^{\prime} \cdots f^{\lambda} U\right)}{(2 \nu) !(\lambda-2 \nu) !\left[(\lambda-1) !(n-\lambda) ! \Delta^{n-\lambda} U\right]^{\nu+1}}
$$

If $2 \nu=\lambda,(59)$ becomes

$$
K_{\lambda}=\frac{\left((f a U)^{\prime} \cdots(f a U)^{\lambda} U\right)\left(f^{\prime} \cdots f^{\lambda} U\right)}{\lambda\left[(\lambda-1) !(n-\lambda) ! \Delta^{n-\lambda} U\right]^{(\lambda+2) / 2}},
$$

which agrees with Maschke's form, K.-G. C. (60). The symbols $f$ and $a$ belong to the quadratic form (3), expressing the length element of $R_{n}$. Further, $(f a U)^{2}=\left(f^{i} a^{2} \cdots a^{\lambda} U^{\lambda+1} \cdots U^{n}\right)$, in which $f^{i}$ is equal to $f^{\imath}$ in $\left(f^{\prime} \cdots f^{\lambda} U\right)$, while the sets of symbols $a^{2} \cdots a^{\lambda}$ are equal in any two consecutive brackets $(f a U)^{2 k-1},(f a U)^{2 k}$ and otherwise distinct.

The result is then that $K_{2 v}$, for the space $R_{\lambda}$, is expressible rationally in terms of the coefficients of the first fundamental form of $R_{n}$ and their derivatives, together with the functions $U^{\lambda+1}, \ldots, U^{n}$ (which define $R_{\lambda}$ in $R_{n}$ ) and their derivatives.

\section{§3. Expression for $K_{\lambda}^{2}$ when $\lambda$ is odd.*}

The invariant $K_{\lambda}^{2}(\lambda$ odd) can be expressed in a manner similar to the above. Substituting from (57) into (54), one gets

$$
\begin{aligned}
& G_{\lambda}^{2}=\omega^{4}\left(\omega(f c U)^{\prime}, \omega(f a U)^{2}, \cdots, \omega(f a U)^{\lambda} U\right) \\
& \quad \times\left(\omega(\phi c U)^{\prime}, \omega(\phi b U)^{2}, \ldots, \omega(\phi b U)^{\lambda} U\right)\left(f^{\prime} \phi^{\prime} f^{3} \cdots f^{\lambda} U\right)\left(f^{2} \phi^{2} \cdots \phi^{\lambda} U\right) .
\end{aligned}
$$

By D. P. (4),

$$
\begin{aligned}
& \left(\omega(f c U)^{\prime}, \omega(f a U)^{2}, \cdots, \omega(f a U)^{\lambda} U^{\top}\right)=\omega^{\lambda}\left((f c U)^{\prime}(f a U)^{2} \cdots(f a U)^{\lambda} U\right) \\
& \quad+\omega^{\lambda-1}(f c U)^{\prime}\left(\omega,(f a U)^{2} \cdots(f a U)^{\lambda} U\right) \\
& \quad+\omega^{\lambda-1} \sum_{i=2}^{\lambda}(f a U)^{i}\left((f c U)^{\prime}(f a U)^{2} \cdots(f a U)^{i-1}, \omega,(f a U)^{i+1} \cdots(f a U)^{\wedge} U\right) \\
& \equiv \omega^{\lambda} \alpha_{1}+\omega^{\lambda-1} \alpha_{2}+\omega^{\lambda-1} \alpha_{3} . \\
& \left(\omega(\phi c U)^{\prime}, \omega(\phi b U)^{2}, \cdots, \omega(\phi b U)^{\lambda} U\right)=\omega^{\lambda}\left((\phi c U)^{\prime}(\phi b U)^{2} \cdots(\phi b U)^{\lambda} U\right) \\
& \quad+\omega^{\lambda-1}(\phi c U)^{\prime}\left(\omega,(\phi b U)^{2} \cdots(\phi b U)^{\lambda} U\right) \\
& \quad+\omega^{\lambda-1} \sum_{k=2}^{\lambda}(\phi b U)^{k}\left((\phi c U)^{\prime}(\phi b U)^{2} \cdots(\phi b U)^{k-1}, \omega,(\phi b U)^{k+1} \cdots(\phi b U)^{\lambda} U\right) \\
& \equiv \omega^{\lambda} \beta_{1}+\omega^{\lambda-1} \beta_{2}+\omega^{\lambda-1} \beta_{3} .
\end{aligned}
$$

* See K.-G. C., p. 93. 
The notations $\alpha_{1}, \alpha_{2}, \alpha_{3}, \beta_{1}, \beta_{2}, \beta_{3}$ are used for brevity to represent the expressions whose relative places they occupy. If we also use

then

$$
\gamma=\left(f^{\prime} \phi^{\prime} f^{3} \cdots f^{\lambda} U\right), \quad \delta=\left(f^{2} \phi^{2} \cdots \phi^{\lambda} U\right),
$$

$$
G_{\lambda}^{2}=\omega^{2 \lambda+2}\left[\omega \alpha_{1}+\alpha_{2}+\alpha_{3}\right]\left[\omega \beta_{1}+\beta_{2}+\beta_{3}\right] \gamma \delta .
$$

The nine terms of this product (omitting powers of $\omega$ ) will now be considered in the following order :
1) $\alpha_{1} \beta_{1} \gamma \delta$,
4) $\alpha_{3} \beta_{2} \gamma \delta$,
7) $\alpha_{1} \beta_{3} \gamma \delta$,
2) $\alpha_{1} \beta_{2} \gamma \delta$
5) $\alpha_{2} \beta_{1} \gamma \delta$,
8) $\alpha_{3} \beta_{3} \gamma \delta$,
3) $\alpha_{2} \beta_{2} \gamma \delta$,
6) $\alpha_{2} \beta_{3} \gamma \delta$,
9) $\alpha_{3} \beta_{1} \gamma \delta$.

For the first we have $\alpha_{1} \beta_{1} \gamma \delta=L$, where

$$
\begin{aligned}
L= & \left((f c U)^{\prime}(f a U)^{2} \cdots(f a U)^{\lambda} U\right) \\
& \times\left((\phi c U)^{\prime}(\phi b U)^{2} \cdots(\phi b U)^{\lambda} U\right)\left(f^{\prime} \phi^{\prime} f^{3} \cdots f^{\lambda} U\right)\left(f^{2} \phi^{2} \cdots \phi^{\lambda} U\right) .
\end{aligned}
$$

The second is shown to vanish as follows :

2) $\alpha_{1} \beta_{2} \gamma \delta=\left(f^{\prime} \phi^{\prime} f^{3} \cdots f^{\lambda} U\right)\left(\phi^{\prime} c^{2} \cdots c^{\lambda} U\right)$

$$
\begin{aligned}
& \times\left(\left(f c^{\top}\right.\right. \\
& \left.(f a U)^{\lambda} U\right)\left(f^{2} \phi^{2} \cdots \phi^{\lambda} U\right)\left(\omega,(\phi b U)^{2} \cdots(\phi b U)^{\lambda} U\right)
\end{aligned}
$$

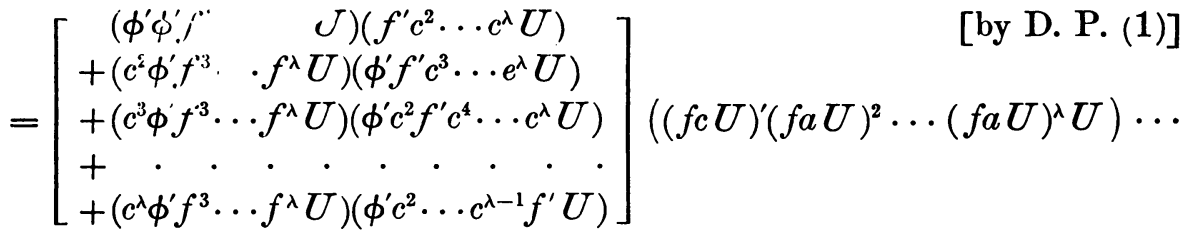

$$
\begin{aligned}
& =(1-\lambda)\left(f^{\prime} \phi^{\prime} f^{3} \ldots f^{\lambda} U\right)\left(\phi^{\prime} c^{2} \cdots c^{\lambda} U\right)\left((f c U)^{\prime}(f a U)^{2} \cdots(f a U)^{\lambda} U\right) \cdots \\
& =(1-\lambda) \alpha_{1} \beta_{2} \gamma \delta \text {. }
\end{aligned}
$$

Hence the second vanishes. The third and fourth are shown to vanish by applying D. P. (1) to exactly the same expressions.

For the fifth term,

5) $\alpha_{2} \beta_{1} \gamma \delta=\left(f^{\prime} \phi^{\prime} f^{3} \cdots f^{\lambda} U\right)\left(f^{\prime} c^{2} \cdots c^{\lambda} U\right)\left((\phi c U)^{\prime}(\phi b U)^{2} \cdots(\phi b U)^{\lambda} U\right)$

$$
\begin{array}{r}
\times\left(\omega,(f a U)^{2} \cdots(f a U)^{\lambda} U\right)\left(f^{2} \phi^{2} \cdots \phi U\right) \\
=\left(\phi^{\prime} f^{3} \cdots f^{\lambda} f^{\prime} U\right)\left(f^{\prime} c^{2} \cdots c^{\lambda} U\right)\left((\phi c U)^{\prime}(\phi b U)^{2} \cdots(\phi b U)^{\lambda} U\right) \cdots
\end{array}
$$

By applying D. P. (1) to the first two forms and simplifying as for 2), we find

$$
\alpha_{2} \beta_{1} \gamma \delta=(1-\lambda) \alpha_{2} \beta_{1} \gamma \delta
$$


Hence 5) vanishes, and the sixth term is shown to vanish by applying D. P. (1) to the same forms.

For the seventh term,

7) $\quad \alpha_{1} \beta_{3} \gamma \delta=\sum_{k=2}^{\lambda}\left(\phi^{k} b^{2} \ldots b^{\lambda} U\right)\left(f^{2} \phi^{2} \ldots \phi^{\lambda} U\right)$

$$
\begin{aligned}
& \times\left((\phi c U)^{\prime}(\phi b U)^{2} \cdots(\phi b U)^{k-1}, \omega, \cdots\right) \\
& \times\left(f^{\prime} \phi^{\prime} f^{3} \cdots f^{\lambda} U\right)\left((f c U)^{\prime}(f a U)^{2} \cdots(f a U)^{\lambda} U\right) .
\end{aligned}
$$

This sum is shown to vanish for all values of $k$ by the method used for (58). and the vanishing of 8 ) follows by the same method.

For the last term,

9) $\alpha_{3} \beta_{1} \gamma \delta=\sum_{i=2}^{\lambda}(f a U)^{i}\left(f^{\prime} \phi^{\prime} f^{3} \cdots f^{\lambda} U\right)$

$$
\begin{aligned}
& \times\left((f c U)^{\prime}(f a U)^{2} \cdots(f a U)^{i-1}, \omega,(f a U)^{i+1} \cdots(f a U)^{\lambda} U\right) \\
& \times\left(f^{2} \phi^{2} \cdots \phi^{\lambda} U\right)\left((\phi c U)^{\prime}(\phi b U)^{2} \cdots(\phi b U)^{\lambda} U\right) .
\end{aligned}
$$

The terms in which $i>3$ vanish by the methods used for (58), but the terms $T_{2}$ (for $i=2$ ) and $T_{3}$ (for $i=3$ ) do not vanish and require special treatment.

We have $T_{2}=\left(f^{3} \cdots f^{\lambda} f^{\prime} \phi^{\prime} U\right)\left(f^{2} a^{2} \cdots a^{\lambda} U\right) N$, where

$$
\begin{aligned}
& N=\left((f c U)^{\prime}, \omega,(f a U)^{3} \cdots(f a U)^{\lambda} U\right)\left(f^{2} \phi^{2} \cdots \phi^{\lambda} U\right) \\
& \left.\times\left((\phi c U)^{\prime}(\phi b U)^{2} \cdots(\phi b U)^{\lambda} U\right)\right),
\end{aligned}
$$

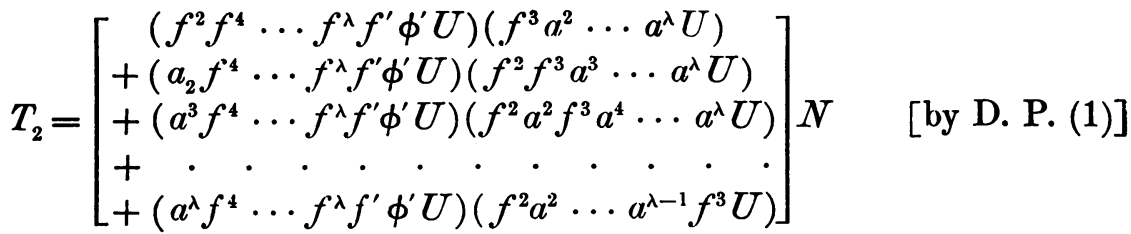

$$
\begin{aligned}
& =\left(f^{2} f^{4} \cdots f^{\lambda} f^{\prime} \phi^{\prime} U\right)\left(f^{3} a^{2} \cdots a^{\lambda} U\right) N+(1-\lambda) T_{2} \text {. }
\end{aligned}
$$

Hence

$$
T_{2}=\frac{1}{\lambda}\left(f^{2} f^{4} \cdots f^{\lambda} f^{\prime} \phi^{\prime} U\right)\left(f^{3} a^{2} \cdots a^{\lambda} U\right) N
$$

If now, we interchange $f^{2}$ and $f^{3}$ and then restore the regular order of symbols, we get

$$
\begin{array}{r}
T_{2}=-\frac{1}{\lambda}\left(f^{\prime} \phi^{\prime} f^{3} \cdots f^{\lambda} U\right)(f a U)^{2}\left((f c U)^{\prime},(f a U)^{2}, \omega,(f a U)^{4} \cdots(f a U)^{\lambda} U\right) \\
\times\left(f^{3} \phi^{2} \cdots \phi^{\lambda} U\right)\left((\phi c U)^{\prime}(\phi b U)^{2} \cdots(\phi b U)^{\lambda} U\right) .
\end{array}
$$

Next, $T_{3}$ may be written as $\left(f^{2} \phi^{2} \ldots \phi^{\lambda} U\right)\left(f^{3} a^{2} \ldots a^{\lambda} U\right) \cdot P$, where 


$$
\begin{aligned}
& P=\left((f c U)^{\prime}(f a U)^{2}, \omega,(f a U)^{4} \cdots(f a U)^{\lambda} U\right) \\
& \times\left(f^{\prime} \phi^{\prime} f^{3} \cdots f^{\lambda} U\right)\left((\phi c U)^{\prime}(\phi b U)^{2} \cdots(\phi b U)^{\lambda} U\right) \cdot
\end{aligned}
$$

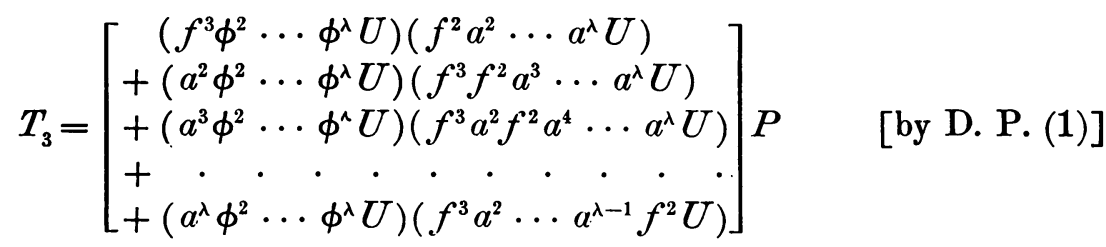

$$
\begin{aligned}
& =\left(f^{3} \phi^{2} \cdots \phi^{\lambda} U\right)\left(f^{2} a^{2} \cdots a^{\lambda} U\right) P+(1-\lambda) T_{3},
\end{aligned}
$$

so that $T_{3}=-T_{2}$.

Thus all nine terms in the second member of (62) vanish except the first, whence

$$
G_{\lambda}^{2}=\omega^{2 \lambda+4} L
$$

Then, by (54) and (56),

$$
K_{\lambda}^{2}=\frac{L}{\lambda\left[(\lambda-1) !(n-\lambda) ! \Delta^{n-\lambda} U\right]^{\lambda+2}},
$$

where $L$ is given by (63), in which the symbols $f, \phi, a, b, c$ belong to the quadratic form (3); the form $(f a U)^{k}=\left(f^{k} a^{2} \ldots a^{\lambda} U^{\lambda+1} \ldots U^{n}\right)$; the $f$ 's (also $\phi$ 's and $c$ 's) with same index are equal; the sets of symbols $a^{2}, \ldots, a^{\lambda}$ (also $\left.b^{2}, \ldots, b^{\lambda}\right)$ are equal in any two consecutive brackets of which the first has even index, and otherwise distinct.

Hence $K_{\lambda}^{2}\left(\lambda\right.$ odd), for the space $R_{\lambda}$, is expressible rationally in terms of the coefficients of the first fundamental form and their derivatives, together with the functions $U^{\lambda+1}, \cdots, U^{n}$ (which define $R_{\lambda}$ in $R_{n}$ ) and their derivatives. 